\title{
The Generalized Ampadu-G Family of Distributions: Properties, Applications and Characterizations
}

\author{
Clement Boateng Ampadu ${ }^{1}$ and Abdulzeid Yen Anafo ${ }^{2}$ \\ ${ }^{1} 31$ Carrolton Road, Boston, MA 02132-6303, USA; e-mail: drampadu@ hotmail.com \\ ${ }^{2}$ University of Mines and Technology, P.O. Box 237, Tarkwa, Ghana, West Africa; \\ e-mail: abdulzeid@aims.edu.gh
}

\begin{abstract}
This paper introduces a new class of distributions called the generalized Ampadu-G (GA$\mathrm{G}$ for short) family of distributions, and with a certain restriction on the parameter space, the family is shown to be a life-time distribution. The shape of the density function and hazard rate function of the GA-G family is described analytically. When $G$ follows the Weibull distribution, the generalized Ampadu-Weibull (GA-W for short) is presented along with its hazard and survival function. Several sub-models of the GA-W family are presented. The transformation technique is applied to this new family of distributions, and we obtain the quantile function of the new family. Power series representations for the cumulative distribution function (CDF) and probability density function (PDF) are also obtained. The $r$ th non-central moments, moment generating function, and Renyi entropy associated with the new family of distributions are derived. Characterization theorems based on two truncated moments and conditional expectation are also presented. A simulation study is also conducted, and we find that using the method of maximum likelihood to estimate model parameters is adequate. The GA-W family of distributions is shown to be practically significant in modeling real life data, and is shown to be superior to some non-trivial generalizations of the Weibull distribution. A further development concludes the paper.
\end{abstract}

Received: March 9, 2020; Accepted: May 6, 2020

2010 Mathematics Subject Classification: 60Exx, 62Exx.

Keywords and phrases: Ampadu-G, Weibull distribution, Poisson distribution, survival function, hazard rate function, statistical properties, characterization theorem, simulation.

Copyright $(9) 2020$ The Authors. This is an open access article distributed under the terms of the Creative Commons Attribution License (http://creativecommons.org/licenses/by/4.0/), which permits unrestricted, use, distribution and reproduction in any medium, or format for any purpose, even commercially provided the work is properly cited. 


\section{Introduction}

Statistical distributions have been developed in recent years by researchers in order to model and predict real world data. There has been a great number of flexible distributions developed by researchers for modelling data. Modelling techniques used are based statistical distributions and this has prompted many researchers in developing new family of distributions through various different methods for defining these families of distributions. Many attempts have been made to propose new families of distributions in the statistical papers by the use of some baseline distributions. Gupta et al. [1] introduced the exponentiated family where the introduction of an extra parameter was introduced. The cumulative distribution function (CDF) of the Gupta et al. [1] proposed exponentiated family is given by,

$$
G(x ; a, \psi)=F(x ; \psi)^{a} \quad a>0, x \in \mathbb{R},
$$

where $\psi$ is a parameter vector of the baseline distribution $G(x ; \psi)$. In relation to [1], many lifetime distributions have been proposed. Some these distributions include, Exponentiated Exponential in Gupta and Kundu [2], Exponentiated beta in Nadarajah [3], exponentiated lognormal in Shirke and Kakade [4], exponentiated Kumaraswamy in Lemonte et al. [5], Exponentiated Power Lindley in Ashour and Eltehiwy [6] and exponentiated Weibull-Pareto in Afify et al. [7]. Another prominent method in this area, is Marshall-Olkin family of distributions proposed by Marshall and Olkin [8] defined by the $\mathrm{CDF}$,

$$
F(x ; \lambda, \psi)=1-\frac{\lambda(1-G(x ; \psi))}{1-(1-\lambda)(1-G(x ; \psi))} \quad \lambda>0, \quad x \in \mathbb{R}
$$

where $\psi$ is a parameter vector of the baseline distribution $G(x ; \psi)$. in relation to [8], many distributions have been generalized. These include, Marshall Olkin extended Weibull of Ghitany et al. [9], Marshall Olkin gamma Marshall Olkin extended logLogistic Weibull of Lepetu et al. [10] and Marshall Olkin gamma Weibull of Saboor and Pogány [11]. Recently, [12] developed the Ampadu-G family of distributions and defined its $\mathrm{CDF}$ as

$$
F(x ; a, \psi)=\frac{1-e^{-a G(x ; \psi)^{2}}}{1-e^{-a}} \quad a>0, x \in \mathbb{R}
$$


where $\psi$ is a parameter vector all of whose entries are positive of the baseline distribution. The corresponding probability density function can be obtained by differentiating the CDF.

This article unfolds as follows. In Section 2, a brief description of the new family of distributions is introduced, where the generalized Ampadu-Weibull family of distributions is presented with sub-models. In Section 3, some statistical properties of the new family are derived. Section 4 presents results of a simulation study. Application of the new family to real-life data is discussed in Section 5, and some characterization theorems are presented in Section 6. The paper concludes with some further developments in Section 7.

\section{Description of the New Family of Distributions}

This section presents the GA-G family of distributions and applies the proposed family of distributions to the Weibull distribution and this new distribution is named the generalized Ampadu-Weibull distribution (GA-W for short). The sub-models of the GA$\mathrm{W}$ distribution are also presented.

\subsection{The GA-G family of distributions}

Definition 2.1. A random variable $X$ is called generalized Ampadu-G distributed if the $\mathrm{CDF}$ is given by

$$
F(x ; \lambda, \beta, \xi)=\frac{1-e^{-\lambda G(x ; \xi)^{\beta}}}{1-e^{-\lambda}}
$$

where $\lambda \in(-\infty, 0) \cup(0, \infty), \quad x \in \mathbb{R}, \quad \xi$ is a vector of parameters in the baseline distribution with $\mathrm{CDF}$, and $\beta>0$.

Proposition 2.2. The PDF of the generalized Ampadu-G family of distributions is given by

$$
f(x ; \lambda, \beta, \xi)=\frac{\lambda \beta e^{\lambda-\lambda G(x ; \xi)^{\beta}} G(x ; \xi)^{\beta-1} g(x ; \xi)}{e^{\lambda}-1},
$$

where $\lambda \in(-\infty, 0) \cup(0, \infty), \quad x \in \mathbb{R}, \quad \xi$ is a vector of parameters in the baseline distribution with $C D F G$ and $P D F g$, and $\beta>0$. 
Remark 2.3. If $\beta=2$, then the Ampadu-G family of distributions [12] can be recovered. Similarly, if $\beta=1$, the $\left(\frac{1}{e}\right)^{\alpha} P T-G$ family of distributions can be recovered [13]. In both instances the parameter space is a subspace of the generalized Ampadu-G family of distributions

\subsection{Physical interpretation of the GA-G family}

Suppose in Definition 2.1, the parameter space of $\lambda$ is restricted to $(0, \infty)$. Let $M$ follow zero truncated Poisson distribution with probability mass function

$$
\mathbb{P}(M=m)=\frac{\lambda^{m}}{m !\left(e^{\lambda}-1\right)}, \quad m=1,2, \ldots ., \lambda>0
$$

Let the failure of each subsystem follow the exponentiated distribution with CDF given by

$$
G(x ; \xi)^{\beta}
$$

where $x \in \mathbb{R}, \beta>0$ and $\xi$ is a vector of parameters in the baseline distribution with CDF $G$. If $Z_{j}$ is the failure time of the $j$ th subsystem and $X$ represents the time to failure of the first out of the $M$ operating subsystems such that $X=\min \left\{Z_{1}, \ldots, Z_{M}\right\}$. Then the conditional CDF of $X$ given $M$ can be shown to be given by

$$
\begin{aligned}
F(x \mid M=m) & =1-\mathbb{P}(X>x \mid M) \\
& =1-\mathbb{P}\left(Z_{1}>x, \ldots, Z_{M}>x\right) \\
& =1-\left[\mathbb{P}\left(Z_{1}>x\right)\right]^{m} \\
& =1-\left[1-\mathbb{P}\left(Z_{1}<x\right)\right]^{m} \\
& =1-\left[1-G(x ; \xi)^{\beta}\right]^{m}, x \in \mathbb{R} .
\end{aligned}
$$

Thus, the marginal CDF of $X$ is given by

$$
F(x)=\frac{1}{e^{\lambda}-1} \sum_{m=1}^{\infty} \frac{\lambda^{m}}{m !}\left\{1-\left[1-G(x ; \xi)^{\beta}\right]^{m}\right\}
$$




$$
=\frac{1-e^{-\lambda G(x ; \xi)^{\beta}}}{1-e^{-\lambda}}, x \in \mathbb{R}
$$

where $\lambda \in(0, \infty)$, and $\xi$ is a vector of parameters in the baseline distribution with CDF $G$, and $\beta>0$. With this physical interpretation, and restriction on the parameter $\lambda$, the generalized Ampadu-G becomes the Poisson Exponentiated $G$ family of distributions. The PDF of the Poisson Exponentiated G family of distributions can be obtained by differentiating the CDF immediately above.

\subsection{Shape of density and hazard function of the GA-G family}

The shapes of the density function and hazard rate function of the GA-G family can be described analytically. The critical points of the density function can be shown to be roots of the equation $\frac{\partial \ln (f(x ; \lambda, \beta, \xi))}{\partial x}=0$ or equivalently

$$
\lambda \beta G(x ; \xi)^{\beta-1} \frac{\partial G(x ; \xi)}{\partial x}-\frac{\beta-1}{G(x ; \xi)} \frac{\partial G(x ; \xi)}{\partial x}-\frac{1}{g(x ; \xi)} \frac{\partial G(x ; \xi)}{\partial x}=0 .
$$

The above equation may have more than one root. If $x=x_{0}$ is a root of the above equation, then it corresponds to a local maximum if $\frac{\partial^{2} \ln (f(x ; \lambda, \beta, \xi))}{\partial^{2} x}<0$, a local minimum if $\frac{\partial^{2} \ln (f(x ; \lambda, \beta, \xi))}{\partial^{2} x}>0, \quad$ and $\quad$ a point $\quad$ of inflection if $\frac{\partial^{2} \ln (f(x ; \lambda, \beta, \xi))}{\partial^{2} x}=0$.

It can be shown that the hazard rate function of the GA-G family is given by

$$
h(x ; \lambda, \beta, \xi)=\frac{\lambda \beta e^{\lambda} g(x ; \xi) G(x ; \xi)^{\beta-1}}{e^{\lambda}-e^{\lambda G(x ; \xi)^{\beta}}}
$$

where the parameter space is the same as in the GA-G family. In a similar way the critical points of the hazard function can be shown to be roots of the equation $\frac{\partial \ln (h(x ; \lambda, \beta, \xi))}{\partial x}=0$ or equivalently 


$$
\frac{1}{g(x ; \xi)} \frac{\partial g(x ; \xi)}{\partial x}+\frac{\beta-1}{G(x ; \xi)} \frac{\partial G(x ; \xi)}{\partial x}+\frac{\lambda \beta e^{\lambda G(x ; \xi)^{\beta}} G(x ; \xi)^{\beta-1} \partial G(x ; \xi)}{e^{\lambda}-e^{\lambda G(x ; \xi)^{\beta}}} \frac{\partial G(x ; \xi)}{\partial x}=0 .
$$

The above equation may have more than one root. If $x=x_{0}$ is a root of the above equation, then it corresponds to a local maximum if $\frac{\partial^{2} \ln (h(x ; \lambda, \beta, \xi))}{\partial^{2} x}<0$, a local minimum if $\frac{\partial^{2} \ln (h(x ; \lambda, \beta, \xi))}{\partial^{2} x}>0$, and a point of inflection if $\frac{\partial^{2} \ln (h(x ; \lambda, \beta, \xi))}{\partial^{2} x}=0$.

\subsection{The GA-W family of distributions}

We apply the generalized Ampadu-G family to the Weibull distribution and this new class of distributions is called the generalized Ampadu-Weibull family of distribution.

Definition 2.4. A random variable $X$ is called generalized Ampadu-Weibull distributed if the $\mathrm{CDF}$ is given by

$$
F(x ; \lambda, \beta, a, b)=\frac{e^{\lambda}-e^{\lambda-\lambda\left(1-e^{-\left(\frac{x}{b}\right)^{a}}\right)^{\beta}}}{e^{\lambda}-1}
$$

where $\lambda \in(-\infty, 0) \cup(0, \infty), \beta, x, a, b>0$.

Proposition 2.5. The PDF of the generalized Ampadu-Weibull distribution is given by,

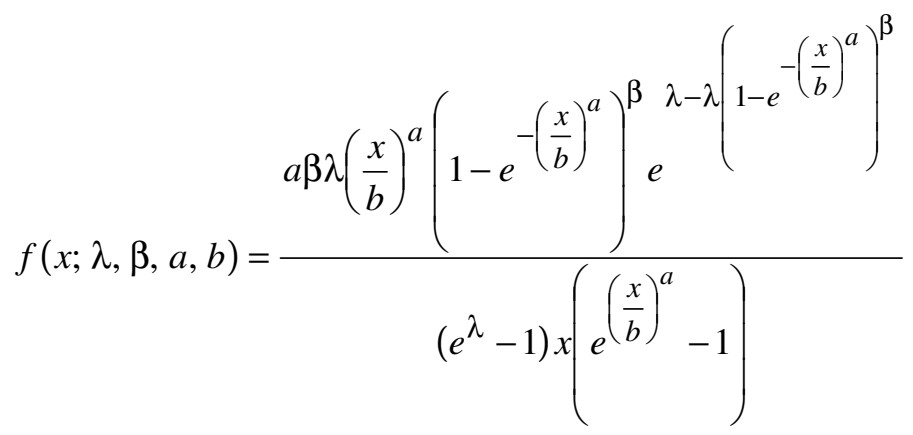

for $x, a, b, \beta>0$, and $\lambda \in(-\infty, 0) \cup(0, \infty)$. 
Proposition 2.6. Let $x, a, b, \beta>0$, and $\lambda \in(-\infty, 0) \cup(0, \infty)$. The survival function and hazard rate function of the generalized Ampadu-Weibull distribution is given by,

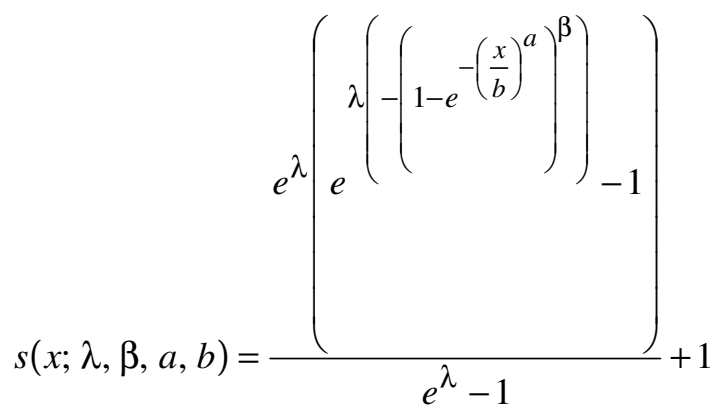

and

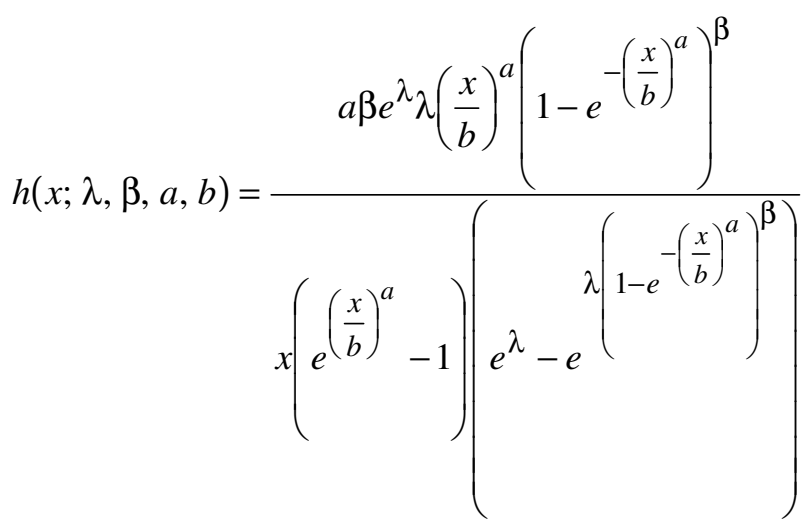

respectively.

Using the estimated parameters in the GA-W distribution recorded in Table 1, we display the survival function and hazard rate function in Appendix $\mathrm{H}$

\subsection{Sub-models of the GA-W family of distributions}

Several families can be derived from the generalized Ampadu-Weibull distribution for different choice of the parameters, and we list some of them below

(i) When $\beta=a=1$ the generalized Ampadu-Weibull distribution reduces to the $\left(\frac{1}{e}\right)^{\alpha} P T$-Exponential distribution with the following CDF; 


$$
F(x ; \lambda, b)=\frac{e^{\lambda}-e^{\lambda e^{-\frac{\pi}{b}}}}{e^{\lambda}-1}
$$

for $x, b>0$, and $\lambda \in(-\infty, 0) \cup(0, \infty)$.

(ii) When $\beta=b=1$ the generalized Ampadu-Weibull distribution reduces to the $\left(\frac{1}{e}\right)^{\alpha} P T$-Standard Exponential Distribution

$$
F(x ; \lambda)=\frac{e^{\lambda}-e^{\lambda e^{-x}}}{e^{\lambda}-1}
$$

for $x>0$, and $\lambda \in(-\infty, 0) \cup(0, \infty)$.

(iii) When $\beta=b=1$ the generalized Ampadu-Weibull distribution reduces to the $\left(\frac{1}{e}\right)^{\alpha} P T$-Standard Exponential Exponentiated Uniform Distribution

$$
F(x ; \lambda, a)=\frac{e^{\lambda}-e^{\lambda e^{-x^{a}}}}{e^{\lambda}-1}
$$

for $x, a>0, \lambda \in(-\infty, 0) \cup(0, \infty)$.

(iv) When $1 \neq \beta>0, a=1$ the generalized Ampadu-Weibull distribution reduces to the generalized Ampadu-Exponential Distribution

$$
F(x ; \lambda, b, \beta)=\frac{e^{\lambda}-e^{\lambda-\lambda\left(1-e^{-\frac{x}{b}}\right)^{\beta}}}{e^{\lambda}-1}
$$

for $x, b, \beta>0$, and $\lambda \in(-\infty, 0) \cup(0, \infty)$.

(v) When $1 \neq \beta>0, a=b=1$ the generalized Ampadu-Weibull distribution reduces to the generalized Ampadu-Standard Exponential Distribution

$$
F(x ; \lambda, \beta)=\frac{e^{\lambda}-e^{\lambda-\lambda(\sinh (x)-\cosh (x)+1)^{\beta}}}{e^{\lambda}-1}
$$

for $x, 1 \neq \beta>0$, and $\lambda \in(-\infty, 0) \cup(0, \infty)$. 
(vi) When $1 \neq \beta>0, b=1$ the generalized Ampadu-Weibull distribution reduces to the generalized Ampadu-Standard Exponential Exponentiated Uniform Distribution

$$
F(x ; \lambda, a, \beta)=\frac{e^{\lambda}-e^{\lambda-\lambda\left(1-e^{-x^{a}}\right)^{\beta}}}{e^{\lambda}-1}
$$

for $x, a, 1 \neq \beta>0$, and $\lambda \in(-\infty, 0) \cup(0, \infty)$.

\section{Statistical Properties}

\subsection{Transformation of random variables}

Theorem 3.1. If $U$ is uniform on $(0,1)$, then

$$
X=\left(\frac{1}{\lambda} \ln \left(\frac{1}{1-U\left(1-e^{-\lambda}\right)}\right)\right)^{\frac{1}{\beta}}
$$

follows the generalized Ampadu-Standard Uniform family of distributions, where

$$
\lambda \in \mathbb{R}-\{0\}
$$

and $\beta>0$.

\subsection{Quantile function}

Theorem 3.2. If $0<p<1$, then

$$
Q(p)=\left(\frac{1}{\lambda} \ln \left(\frac{1}{1-p\left(1-e^{-\lambda}\right)}\right)\right)^{\frac{1}{\beta}}
$$

is the quantile function of the generalized Ampadu-Standard Uniform family of distributions, where

$$
\lambda \in \mathbb{R}-\{0\}
$$

and $\beta>0$.

For some choices of $p \in(0,1), \lambda$ and $\beta$, we display $Q(p)$ for the generalized Ampadu-Standard Uniform distribution in Appendix $F$ 


\subsection{Expansion of CDF}

Let $r$ be arbitrary positive integer, by the Binomial Theorem, we can write

$$
\left(1-e^{-\lambda G(x ; \xi)^{\beta}}\right)^{r}=\sum_{k=0}^{r}\left(\begin{array}{l}
r \\
k
\end{array}\right)(-1)^{r} e^{-\lambda r G(x ; \xi)^{\beta}} .
$$

By the power series representation for the exponential function, we can write

$$
e^{-\lambda r G(x ; \xi)^{\beta}}=\sum_{i=0}^{\infty} \frac{(-1)^{i} \lambda^{i} r^{i} G(x ; \xi)^{\beta i}}{i !} .
$$

Thus if $r=1$, we can write

$$
1-e^{-\lambda G(x ; \xi)^{\beta}}=\sum_{k=0}^{1}\left\{\sum_{i=0}^{\infty}\left(\begin{array}{l}
1 \\
k
\end{array}\right) \frac{(-1)^{i+1} \lambda^{i} G(x ; \xi)^{\beta i}}{i !}\right\} .
$$

Since $\left(\begin{array}{l}1 \\ 0\end{array}\right)=\left(\begin{array}{l}1 \\ 1\end{array}\right)=1$, the equation immediately above can be written as

$$
1-e^{-\lambda G(x ; \xi)^{\beta}}=2 \sum_{i=0}^{\infty} \frac{(-1)^{i+1} \lambda^{i} G(x ; \xi)^{\beta i}}{i !} .
$$

Thus we have the following

Proposition 3.3. The CDF of the generalized Ampadu-G family of distributions admit the following expansion

$$
\sum_{i=0}^{\infty} w_{i} G(x ; \xi)^{\beta i},
$$

where $w_{i}=\frac{2(-1)^{1+i} \lambda^{i}\left(1-e^{-\lambda}\right)^{-1}}{i !}, \lambda \in \mathbb{R}-\{0\}, \beta>0, x \in \mathbb{R}$, and $\xi$ is a vector of parameters in the baseline distribution with $C D F G(x ; \xi)$.

\subsection{Expansion of PDF}

Proposition 3.4. The PDF of the generalized Ampadu-G family of distributions admit the following expansion

http://www.earthlinepublishers.com 


$$
\sum_{k=0}^{\infty} \sum_{j=0}^{k} w_{k, j} g(x ; \xi) G(x ; \xi)^{\beta j+\beta-1},
$$

where $w_{k, j}=\left(\begin{array}{c}k \\ j\end{array}\right) \frac{\lambda^{k+1}(-1)^{j} \beta}{k !\left(e^{\lambda}-1\right)}, \quad \lambda \in \mathbb{R}-\{0\}, \quad \beta>0, \quad x \in \mathbb{R}, \quad$ and $\xi$ is a vector of parameters in the baseline distribution with $C D F G(x ; \xi)$ and PDF $g(x ; \xi)$.

\section{5. $r$ th non-central moment}

Proposition 3.5. The rth non-central moments of the generalized Ampadu-G family of distributions, for $r \in \mathbb{N}$ can be expressed as

$$
\mu_{r}^{\prime}=\sum_{k=0}^{\infty} \sum_{j=0}^{k} w_{k, j} \int_{-\infty}^{\infty} x^{r} g(x ; \xi) G(x ; \xi)^{\beta j+\beta-1} d x,
$$

where $w_{k, j}=\left(\begin{array}{c}k \\ j\end{array}\right) \frac{\lambda^{k+1}(-1)^{j} \beta}{k !\left(e^{\lambda}-1\right)}, \quad \lambda \in \mathbb{R}-\{0\}, \quad \beta>0, \quad x \in \mathbb{R}, \quad$ and $\xi$ is a vector of parameters in the baseline distribution with $C D F G(x ; \xi)$ and PDF $g(x ; \xi)$.

Given a random variable $X$ with PDF $f(x)$, the ordinary moments, for $r \in \mathbb{N}$, are given by

$$
E\left[X^{r}\right]=\int_{-\infty}^{\infty} x^{r} f(x) d x
$$

However, if the random variable $X$ in question has $\operatorname{CDF} F(x)$ and quantile function $Q_{X}$, then after the substitution $u=F(x)$, he ordinary moments can be expressed as

$$
E\left[X^{r}\right]=\int_{0}^{1}\left(Q_{X}(u)\right)^{r} d u
$$

Using this representation we display the first ten moments of the generalized AmpaduStandard Uniform distribution for some choice parameters in Appendix G.

\subsection{Moment generating function}

Proposition 3.6. The moment generating function of the generalized Ampadu-G family of distributions can be expressed as 


$$
M_{X}(t)=\sum_{r, k=0}^{\infty} \sum_{j=0}^{k} \frac{t^{r}}{r !} w_{k, j} \int_{-\infty}^{\infty} x^{r} g(x ; \xi) G(x ; \xi)^{\beta j+\beta-1} d x
$$

where $w_{k, j}=\left(\begin{array}{l}k \\ j\end{array}\right) \frac{\lambda^{k+1}(-1)^{j} \beta}{k !\left(e^{\lambda}-1\right)}, \quad \lambda \in \mathbb{R}-\{0\}, \quad \beta>0, \quad x \in \mathbb{R}$, and $\xi$ is a vector of parameters in the baseline distribution with CDF $G(x ; \xi)$ and PDF $g(x ; \xi)$.

\subsection{Renyi entropy}

Proposition 3.7. The Renyi entropy of the generalized Ampadu-G family of distributions can be expressed as

$$
I_{R}(\delta)=\frac{1}{1-\delta} \log \left[\left(\frac{\lambda \beta e^{\lambda}}{e^{\lambda}-1}\right)^{\delta} \sum_{k=0}^{\infty} w_{k} \int_{-\infty}^{\infty} g(x ; \xi)^{\delta} G(x ; \xi)^{\beta k+\delta(\beta-1)} d x\right],
$$

where $w_{k}=\frac{(-1)^{k} \delta^{k} \lambda^{k}}{k !}, 1 \neq \delta>0, \lambda \in \mathbb{R}-\{0\}, \beta>0, x \in \mathbb{R}$, and $\xi$ is a vector of parameters in the baseline distribution with CDF $G(x ; \xi)$ and PDF $g(x ; \xi)$.

\section{Simulation Study}

In this simulation study samples of sizes $400,550,700$, and 900, are drawn from the generalized Ampadu-Weibull family of distributions. The samples have been drawn for $(a, b, \lambda, \beta)=(1.4,2.2,2.2,1.9)$ in the first simulation study, and the average bias and root mean square error are obtained. The procedure has been repeated 200 times and the results are summarized in Appendix A. In the second simulation study the samples have been drawn for $(a, b, \lambda, \beta)=(1.4,1.4,1.4,1.9)$ and the average estimates and mean square error of the parameters are obtained. The procedure has been repeated 200 times and the results are summarized in Appendix B.

From Appendix A, we find that the average bias is around zero and hence the estimation method is adequate. We have also observed that the estimated root mean square errors (RMSE) consistently decrease with increasing sample size as seen in Appendix C. From Appendix B, we find that the average estimates are consistently close to the true values, and hence the estimation method is adequate. We have also observed 
that the estimated mean square errors (MSE) consistently decrease with increasing sample size as seen in Appendix D.

Overall, the simulation study conducted indicates that using the method of maximum likelihood to estimates the parameters in the generalized Ampadu-G family of distributions is adequate.

\section{Application}

\subsection{Maximum likelihood estimation in the GA-G distribution}

In this section, we obtain the maximum likelihood estimators (MLEs) for the parameters in the generalized Ampadu-G family of distributions. For this, let $X_{1}, X_{2}, \ldots ., X_{n}$ be a random sample of size $n$ from the generalized Ampadu-G family of distributions. The likelihood function from Proposition 2.2 is given by

$$
L=\prod_{i=1}^{n}\left(\frac{\lambda \beta e^{\lambda-\lambda G\left(x_{i} ; \xi\right)^{\beta}} G\left(x_{i} ; \xi\right)^{\beta-1} g\left(x_{i} ; \xi\right)}{e^{\lambda}-1}\right) .
$$

From the above the log-likelihood function is given by

$$
\begin{aligned}
\ln L= & n(\ln \lambda+\ln \beta+\lambda)-\sum_{i=1}^{n} \lambda G\left(x_{i} ; \xi\right)^{\beta}+(\beta-1) \sum_{i=1}^{n} \ln \left(G\left(x_{i} ; \xi\right)\right) \\
& +\sum_{i=1}^{n} \ln g\left(x_{i} ; \xi\right)-n \ln \left(e^{\lambda}-1\right) .
\end{aligned}
$$

Obtaining the partial derivatives of the equation immediately above, we get the following

$$
\begin{aligned}
& \frac{\partial \ln L}{\partial \lambda}=n\left(\frac{1}{\lambda}+1\right)-\sum_{i=1}^{n} G\left(x_{i} ; \xi\right)^{\beta}-\frac{n e^{\lambda}}{e^{\lambda}-1} \\
& \frac{\partial \ln L}{\partial \beta}=\frac{n}{\beta}-\lambda \beta \sum_{i=1}^{n} G\left(x_{i} ; \xi\right)^{\beta-1} \frac{\partial G\left(x_{i} ; \xi\right)}{\partial \beta}+\sum_{i=1}^{n} \ln \left(G\left(x_{i} ; \xi\right)\right) \\
& \frac{\partial \ln L}{\partial \xi}=\sum_{i=1}^{n} \lambda \beta \frac{\partial G\left(x_{i} ; \xi\right)}{\partial \xi} G\left(x_{i} ; \xi\right)^{\beta-1}+(\beta-1) \sum_{i=1}^{n} \frac{\frac{\partial G(x-\xi)}{\partial \xi}}{G\left(x_{i} ; \xi\right)}+\sum_{i=1}^{n} \frac{\frac{\partial g\left(x_{i} ; \xi\right)}{\partial \xi}}{g\left(x_{i} ; \xi\right)} .
\end{aligned}
$$


By setting the three equations immediately above to zero and solving simultaneously we obtain the maximum likelihood estimates.

\subsection{Performance of GA-W distribution}

In this section we investigate the performance of the generalized Ampadu-Weibull distribution (GA-W) in fitting real-life data. We consider the data-set on the survival times of 72 guinea pigs infected with virulent tubercle bacilli [14]. The performance of the generalized Ampadu-Weibull distribution is compared with two other generalizations of the Weibull distribution, that is, the Marshall-Olkin Weibull (MOW) and the exponentiated Weibull (EW) distributions. The measures of goodness of fit we consider include Akaike information criterion (AIC), Bayesian information criterion (BIC), 2Log-Likelihood, and second-order Akaike Information Criterion (AICc). The Weibull distribution can be written in different forms, however in defining the MOW distribution and the EW distribution, we use the form of the Weibull distribution as employed in Definition 2.4. In particular, using the form of the Weibull distribution in Definition 2.4 in equation (1.1) of [8], and then differentiating, we have the following as the PDF of the MOW distribution

$$
f(x ; a, b, \alpha)=\frac{a \alpha e^{\left(\frac{x}{b}\right)^{a}}\left(\frac{x}{b}\right)^{a}}{x\left(e^{\left(\frac{x}{b}\right)^{a}}+\alpha-1\right)^{2}}
$$

and the following as the PDF of the EW distribution, upon powering a positive real number to the form of the Weibull distribution in Definition 2.4, and then differentiating the result

$$
g(x ; a, b, \gamma)=\frac{a \gamma\left(\frac{x}{b}\right)^{a}\left(1-e^{\left.-\left(\frac{x}{b}\right)^{a}\right)^{\gamma}}\right.}{x\left(e^{\left(\frac{x}{b}\right)^{a}}-1\right)}
$$

http://www.earthlinepublishers.com 
The idea of powering a positive real number (say) to the CDF of any distribution is popular technique in distribution theory, and is due to [1]

Table 1. Estimates for the parameter of fitted distribution

\begin{tabular}{cccc}
\hline Distribution & Parameters & Estimates & Standard error \\
\hline GA-W & $\hat{a}$ & 1.45676 & 0.53782 \\
& $\hat{b}$ & 199.65453 & 105.03858 \\
& $\hat{\lambda}$ & 2.25265 & 1.99160 \\
& $\hat{\beta}$ & 1.92307 & 1.19978 \\
\hline MOW & $\hat{a}$ & 0.61507 & 0.13003 \\
& $\hat{b}$ & 12.97847 & 11.39748 \\
EW & $\hat{\alpha}$ & 102.72953 & 107.15502 \\
& $\hat{a}$ & 1.07124 & 0.29158 \\
& $\hat{b}$ & 99.17756 & 45.47998 \\
& $\hat{\gamma}$ & 3.16194 & 1.92368 \\
\hline
\end{tabular}

The parameter estimates in the GA-W, MOW, and EW distributions along with their standard errors are summarized in Table 1 above. An inspection of Table 2 below shows that the EW distribution is preferred in comparison with the other distributions in fitting the same data. Among the non-trivial generalizations of the Weibull distribution, it can be seen that the GA-W distribution is preferred to the MOW distribution in fitting the same data. The GA-W distribution as a non-trivial generalization of the Weibull distribution should play significant role in fitting real-life data from various disciplines.

Table 2. Goodness-of-fit measures

\begin{tabular}{ccccc}
\hline Distribution & AIC & BIC & AICc & -2Log-Likelihood \\
\hline GA-W & 858.0345 & 867.1412 & 858.6316 & 850.0345 \\
\hline MOW & 858.515 & 865.345 & 858.8679 & 852.515 \\
\hline EW & 857.3916 & 864.2216 & 857.7445 & 851.3916
\end{tabular}

The estimated PDFs to the histogram and the estimated CDFs to the empirical distribution are displayed in Appendix E.

\section{Characterization Theorems}

The characterization of statistical distributions plays a major role in stochastic modeling. In this section we present some characterizations of the generalized Ampadu$\mathrm{G}$ family of distributions. Our first characterization theorem is based on a simple 
relationship between two truncated moments, and for related works in this direction, the reader is referred to [15]-[20].

At first, we recall the following which will be useful in the sequel

Theorem 6.1. [16] Let $(\Omega, \Sigma, \mathbb{P})$ be a given probability space, and let $I=[a, b]$ be an interval for some $a<b(a=-\infty, b=\infty$ might as well be allowed). Let $X: \Omega \mapsto I$ be a continuous random variable with probability distribution function $F$, and let $q_{1}$ and $q_{2}$ be two real functions on I such that

$$
\mathbb{E}\left[q_{1}(X) \mid X \geq x\right]=\mathbb{E}\left[q_{2}(X) \mid X \geq x\right] \eta(x), x \in I,
$$

is defined with some real function $\eta$. Assume that $q_{1}, q_{2} \in C^{1}(I)$, and $\eta \in C^{2}(I)$, and $F$ is twice continuously differentiable and strictly monotone increasing on the set $I$. Finally, assume that the equation $n q_{2}=q_{1}$ has no real solutions in the interior of $I$. Then $F$ is uniquely determined by the functions $q_{1}, q_{2}$, $\eta$. In particular,

$$
F(x)=\int_{a}^{x} C\left|\frac{\eta^{\prime}(u)}{\eta(u) q_{2}(u)-q_{1}(u)}\right| \exp (-s(u)) d u,
$$

where the function s is a solution of the differential equation

$$
s^{\prime}=\frac{\eta^{\prime} q_{2}}{\eta q_{2}-q_{1}}
$$

and $C$ is a constant chosen to make $\int_{I} d F=1$.

Remark 6.2. The characterization based on the ratio of two truncated moments is stable in the sense of weak convergence, and for more details see [21]

Our second characterization result employs a single function $\psi$ of $X$ and states a characterization result in terms of $\psi(X)$. The following known results is useful for our purposes here.

Proposition 6.3. [22] Let $X: \Omega \mapsto(a, b)$ be a continuous random variable with CDF F. Let $\psi(x)$ be a differentiable function on $(a, b)$ with $\lim _{x \rightarrow a^{+}} \psi(x)=\delta>1$ and

$$
\lim _{x \rightarrow b^{-}} \psi(x)=\infty
$$


Then

$$
\mathbb{E}\left[(\psi(X))^{\delta} \mid X \leq x\right]=\delta(\psi(x))^{\delta-1}, x \in(a, b),
$$

implies

$$
\psi(x)=\delta\left[1-(F(x))_{1-\delta}^{1}\right]^{-1}, x \in(a, b) .
$$

Proposition 6.4. [22] Let $X: \Omega \mapsto(a, b)$ be a continuous random variable with CDF F. Let $\psi_{1}(x)$ be a differentiable function on $(a, b)$ with $\lim _{x \rightarrow a^{+}} \psi_{1}(x)=\frac{\delta}{2}>\frac{1}{2}$ and

$$
\lim _{x \rightarrow b^{-}} \psi_{1}(x)=\infty
$$

Then

$$
\mathbb{E}\left[\left(\psi_{1}(X)\right)^{\delta} \mid X \leq x\right]=\delta\left(\psi_{1}(x)\right)^{\delta-1}, x \in(a, b),
$$

implies

$$
\psi_{1}(x)=\delta\left[1+(F(x))^{\frac{1}{1-\delta}}\right]^{-1}, x \in(a, b) .
$$

\subsection{Characterization based on two truncated moments}

The main result here is the following

Proposition 6.5. Let $X: \Omega \mapsto \mathbb{R}$ be a continuous random variable, and let $q_{2}(x)=1$, and

$$
q_{1}(x)=\frac{1-e^{-\lambda G(x ; \xi)^{\beta}}}{1-e^{-\lambda}}
$$

then the PDF of $X$ is

$$
\frac{\left.\lambda \beta e^{\lambda-\lambda G(x ; \xi)}\right)^{\beta} G(x ; \xi)^{\beta-1} g(x ; \xi)}{e^{\lambda}-1}
$$

iff the function $\eta$ in Theorem 6.1 has the form 


$$
\eta(x)=\frac{1-e^{-\lambda G(x ; \xi)^{\beta}}}{2\left(1-e^{-\lambda}\right)}
$$

where $\lambda \in(-\infty, 0) \cup(0, \infty), x \in \mathbb{R}, \xi$ is a vector of parameters in the baseline distribution with $C D F G$ and $P D F g$, and $\beta>0$.

Proof. Let $X$ have PDF

$$
\frac{\lambda \beta e^{\lambda-\lambda G(x ; \xi)^{\beta}} G(x ; \xi)^{\beta-1} g(x ; \xi)}{e^{\lambda}-1}
$$

then for all $x \in \mathbb{R}$ we deduce the following

$$
(1-F(x)) \mathbb{E}\left[q_{2}(X) \mid X \geq x\right]=\frac{1-e^{-\lambda G(x ; \xi)^{\beta}}}{1-e^{-\lambda}}
$$

and

$$
(1-F(x)) \mathbb{E}\left[q_{1}(X) \mid X \geq x\right]=\frac{\left(1-e^{\left.-\lambda G(x ; \xi)^{\beta}\right)^{2}}\right.}{2\left(1-e^{-\lambda}\right)^{2}}
$$

and finally

$$
\begin{aligned}
& \frac{1-e^{-\lambda G(x ; \xi)^{\beta}}}{2\left(1-e^{-\lambda}\right)}-\frac{1-e^{-\lambda G(x ; \xi)^{\beta}}}{1-e^{-\lambda}} \\
= & -\frac{1-e^{-\lambda G(x ; \xi)^{\beta}}}{2\left(1-e^{-\lambda}\right)} \\
< & 0 .
\end{aligned}
$$

Conversely, if

$$
\eta(x)=\frac{1-e^{-\lambda G(x ; \xi)^{\beta}}}{2\left(1-e^{-\lambda}\right)}
$$

then we can check that

$$
s^{\prime}(x)=-\frac{\frac{\lambda \beta e^{\lambda-\lambda G(x ; \xi)^{\beta}} G(x ; \xi)^{\beta-1} g(x ; \xi)}{e^{\lambda}-1}}{\frac{1-e^{-\lambda G(x ; \xi)^{\beta}}}{1-e^{-\lambda}}}
$$

http://www.earthlinepublishers.com 
and hence

$$
s(x)=-\log \left(\frac{1-e^{-\lambda G(x ; \xi)^{\beta}}}{1-e^{-\lambda}}\right) .
$$

Now in view of Theorem 6.1, $X$ has PDF

$$
\frac{\lambda \beta e^{\lambda-\lambda G(x ; \xi)^{\beta}} G(x ; \xi)^{\beta-1} g(x ; \xi)}{e^{\lambda}-1} .
$$

If $q_{2}$ is given by the previous Proposition, then we have the following

Corollary 6.6. Let $X: \Omega \mapsto \mathbb{R}$ be a continuous random variable. The PDF of $X$ is

$$
\frac{\lambda \beta e^{\lambda-\lambda G(x ; \xi)^{\beta}} G(x ; \xi)^{\beta-1} g(x ; \xi)}{e^{\lambda}-1}
$$

iff there exist functions $q_{1}$ and $\eta$ defined in Theorem 6.1 satisfying the differential equation

$$
\frac{\eta^{\prime}(x)}{\eta(x)-q_{1}(x)}=-\frac{\frac{\lambda \beta e^{\lambda-\lambda G(x ; \xi)^{\beta}} G(x ; \xi)^{\beta-1} g(x ; \xi)}{e^{\lambda}-1}}{\frac{1-e^{-\lambda G(x ; \xi)^{\beta}}}{1-e^{-\lambda}}}
$$

for all $x \in \mathbb{R}$, where $\lambda \in(-\infty, 0) \cup(0, \infty), \xi$ is a vector of parameters in the baseline distribution with CDF $G$ and $P D F g$, and $\beta>0$.

Remark 6.7. The general solution of the differential equation in the above Corollary is given by

$$
\eta(x)=\frac{1-e^{-\lambda}}{1-e^{-\lambda G(x ; \xi)^{\beta}}}\left[\int q_{1}(x) \frac{\lambda \beta e^{\lambda-\lambda G(x ; \xi)^{\beta}} G(x ; \xi)^{\beta-1} g(x ; \xi)}{e^{\lambda}-1} d x+D\right]
$$

for all $x \in \mathbb{R}$, where $\lambda \in(-\infty, 0) \cup(0, \infty), \xi$ is a vector of parameters in the baseline distribution with CDF $G$ and PDF $g, \beta>0$, and $D$ is a constant. One set of appropriate functions is given by the previous Proposition with $D=0$. 


\subsection{Characterization based on conditional expectation}

\subsubsection{Characterization based on Proposition 6.3}

A characterization of the generalized Ampadu-G family of distributions based on the first Proposition is obtained by taking $(a, b)=(0, \infty)$ and

$$
\psi(x)=\delta\left[1+\left(\frac{1-e^{-\lambda G(x ; \xi)^{\beta}}}{1-e^{-\lambda}}\right)^{\frac{1}{\delta-1}}\right]^{-1},
$$

where $\lambda \in(-\infty, 0) \cup(0, \infty), x \in \mathbb{R}, \xi$ is a vector of parameters in the baseline distribution with $\operatorname{CDF} G, \beta>0$, and $\delta>1$.

\subsubsection{Characterization based on Proposition 6.4}

A characterization of the generalized Ampadu-G family of distributions based on the second Proposition is obtained by taking $(a, b)=(0, \infty)$ and

$$
\psi_{1}(x)=\delta\left[1+\left(1-\frac{1-e^{-\lambda G(x ; \xi)^{\beta}}}{1-e^{-\lambda}}\right)^{\frac{1}{\delta-1}}\right]^{-1}
$$

where $\lambda \in(-\infty, 0) \cup(0, \infty), x \in \mathbb{R}, \xi$ is a vector of parameters in the baseline distribution with $\operatorname{CDF} G, \beta>0$, and $\delta>\frac{1}{2}$.

\section{Further Development}

In [23] they introduced a new method of generating continuous distributions based on the alpha power transformation family of [24] by inverting the equation

$$
\frac{\alpha^{F(x)}-1}{\alpha-1}=G(x)
$$

that is, by solving for $F(x)$ in the above equation. In a similar way, we can add to the class of within quantile distributions. As a further development, we propose a so called generalized Ampadu-F within the quantile distribution. This distribution is obtained by 
solving for $G(x ; \xi)$ in Definition 2.1, thus, obtaining

$$
\left\{\frac{1}{\lambda} \ln \left(\frac{1}{1-\left(1-e^{-\lambda}\right) F(x ; \xi)}\right)\right\}^{\frac{1}{\beta}},
$$

where $\lambda \in(-\infty, 0) \cup(0, \infty), x \in \mathbb{R}, \xi$ is a vector of parameters in the baseline distribution with $\mathrm{CDF} F$, and $\beta>0$.

In particular, we ask the reader to investigate some properties and applications of this new class of within quantile distributions.

\section{References}

[1] R. C. Gupta, P. L. Gupta and R. D. Gupta, Modeling failure time data by Lehmann alternatives, Comm. Statist. Theory Methods 27 (1998), 887-904. https://doi.org/10.1080/03610929808832134

[2] R. D. Gupta and D. Kundu, Exponentiated exponential family: an alternative to gamma and Weibull distributions, Biom. J. 43(1) (2001), 117-130. https://doi.org/10.1002/1521-4036(200102)43:1\%3C117::AID-BIMJ117\%3E3.0.CO;2-R

[3] S. Nadarajah, Exponentiated beta distributions, Comput. Math. Appl. 49(7-8) (2005), 1029-1035. https://doi.org/10.1016/j.camwa.2004.11.008

[4] D. T. Shirke and C. S. Kakade, On exponentiated lognormal distribution, International Journal of Agricultural and Statistical Sciences 2(2) (2006), 319-326.

[5] A. J. Lemonte, W. Barreto-Souza and G. M. Cordeiro, The exponentiated Kumaraswamy distribution and its log-transform, Braz. J. Probab. Stat. 27(1) (2013), 31-53. https://doi.org/10.1214/11-BJPS149

[6] S. K. Ashour and M. A. Eltehiwy, Exponentiated power Lindley distribution, Journal of Advanced Research 6(6) (2015), 895-905. https://doi.org/10.1016/j.jare.2014.08.005

[7] A. Z. Afify, H. M. Yousof, G. G. Hamedani and G. R. Aryal, The Exponentiated Weibull-Pareto Distribution with Application, J. Stat. Theory Appl. 15(4) (2016), 326-344. https://doi.org/10.2991/jsta.2016.15.4.2

[8] A. W. Marshall and I. Olkin, A new method for adding a parameter to a family of distributions with application to the exponential and Weibull families, Biometrika 84(3) (1997), 641-652. https://doi.org/10.1093/biomet/84.3.641 
[9] M. E. Ghitany, E. K. Al-Hussaini and R. A. Al-Jarallah, Marshall-Olkin extended Weibull distribution and its application to censored data, J. Appl. Stat. 32(10) (2005), 1025-1034. https://doi.org/10.1080/02664760500165008

[10] L. Lepetu, B. O. Oluyede, B. Makubate, S. Foya and P. Mdlongwa, Marshall-Olkin log-logistic extended Weibull distribution: theory, properties and applications, Journal of Data Science 15 (2017), 691-722.

[11] A. Saboor and T. K. Pogány, Marshall-Olkin gamma-Weibull distribution with applications, Comm. Statist. Theory Methods 45(5) (2016), 1550-1563. https://doi.org/10.1080/03610926.2014.953694

[12] Clement Boateng Ampadu, The Ampadu- $G$ family of distributions with application to the $T-X(W)$ class of distributions, Annal Biostat \& Biomed Appli 1(4) (2019), ABBA.MS.ID.000518. https://doi.org/10.33552/ABBA.2019.01.000518

[13] Clement Boateng Ampadu, The Ampadu APT - $q T-X$ family of distributions induced by $\mathrm{V}$ with an illustration to data in the health sciences, Annal Biostat \& Biomed Appli 2(1) (2019), ABBA.MS.ID.000526. https://doi.org/10.33552/ABBA.2019.02.000526

[14] T. Bjerkedal, Acquisition of resistance in guinea pigs infected with different doses of virulent tubercle bacilli, American Journal of Epidemiology 72(1) (1960), 130-148. https://doi.org/10.1093/oxfordjournals.aje.a120129

[15] W. Glänzel, A. Teles and A. Schubert, Characterization by truncated moments and its application to Pearson-type distributions, Z. Wahrsch. Verw. Gebiete 66(2) (1984), 173-183. https://doi.org/10.1007/BF00531527

[16] W. Glänzel, A characterization theorem based on truncated moments and its application to some distribution families, in: Mathematical Statistics and Probability Theory (pp. 75-84), Springer, Netherlands, 1987. https://doi.org/10.1007/978-94-009-3965-3_8

[17] W. Glanzel, Some consequences of a characterization theorem based on truncated moments, Statistics 21(4) (1990), 613-618. https://doi.org/10.1080/02331889008802273

[18] W. Glänzel and G. G. Hamedani, Characterizations of univariate continuous distributions, Studia Sci. Math. Hungar. 37(1-2) (2001), 83-118. https://doi.org/10.1556/sscmath.37.2001.1-2.5

[19] G. G. Hamedani, Characterizations of univariate continuous distributions, II, Studia Sci. Math. Hungar. 39(3-4) (2002), 407-424. https://doi.org/10.1556/sscmath.39.2002.3-4.11 
[20] G. G. Hamedani, Characterizations of univariate continuous distributions, III, Studia Sci. Math. Hungar. 43(3) (2006), 361-385. https://doi.org/10.1556/sscmath.43.2006.3.4

[21] Morad Alizadeh, Faton Merovci and G. G. Hamedani, Generalized transmuted family of distributions: properties and applications, Hacet. J. Math. Stat. 46(4) (2017), 645667. https://doi.org/10.15672/HJMS.201610915478

[22] Faton Merovci, Morad Alizadeh and G. G. Hamedani, Another generalized transmuted family of distributions: properties and applications, Austrian Journal of Statistics 45 (2016), 71-93. https://doi.org/10.17713/ajs.v45i3.109

[23] M. Nassar, A. Alzaatreh, O. Abo-Kasem, M. Mead and M. Mansoor, A new family of generalized distributions based on alpha power transformation with application to cancer data, Ann. Data. Sci. 5(3) (2018), 421-436. https://doi.org/10.1007/s40745-018-0144-5

[24] A. Mahdavi and D. Kundu, A new method for generating distributions with an application to exponential distribution, Commun. Statist. Theory Methods 46(13) (2017), 6543-6557. https://doi.org/10.1080/03610926.2015.1130839 
Appendix A

Table 3. Result of simulation study I.

\begin{tabular}{ccc}
\hline & \multicolumn{2}{c}{ Parameter $a$} \\
\hline Sample Size & Average Bias & RMSE \\
\hline 400 & 0.06314528 & 0.3069322 \\
\hline 550 & 0.04363775 & 0.2662859 \\
\hline 700 & 0.01180784 & 0.2585421 \\
\hline 900 & 0.01583037 & 0.230548 \\
\hline \multicolumn{3}{c}{ Parameter $b$} \\
\hline Sample Size & Average Bias & RMSE \\
\hline 400 & 0.05867861 & 0.8073855 \\
\hline 550 & -0.04456308 & 0.6677191 \\
\hline 700 & -0.05407836 & 0.6577186 \\
\hline 900 & -0.04024325 & 0.6239734 \\
\hline \multicolumn{3}{c}{ Parameter $\alpha$} \\
\hline Sample Size & Average Bias & RMSE \\
\hline 400 & -0.2699235 & 1.984697 \\
\hline 550 & -0.5178007 & 2.290093 \\
\hline 700 & -0.3122277 & 1.704197 \\
\hline 900 & -0.2333839 & 1.530067 \\
\hline \multicolumn{3}{c}{ Parameter $\beta$} \\
\hline Sample Size & Average BIas & RMSE \\
\hline 400 & -0.5177613 & 1.642966 \\
\hline 550 & -0.3030561 & 0.9876225 \\
\hline 700 & -0.2159831 & 0.927763 \\
\hline 900 & -0.1857769 & 0.9006777 \\
\hline \multicolumn{3}{c}{}
\end{tabular}


Appendix B

Table 4. Result of simulation study II

\begin{tabular}{ccc}
\hline \multicolumn{3}{c}{ Parameter $\beta$} \\
\hline Sample Size & Average Estimate & MSE \\
\hline 400 & 2.049654 & 0.5738651 \\
\hline 550 & 1.935658 & 0.420398 \\
\hline 700 & 1.955605 & 0.4073591 \\
\hline 900 & 1.951792 & 0.318611 \\
\hline \multicolumn{3}{c}{ Parameter $a$} \\
\hline Sample Size & Average Estimate & MSE \\
\hline 400 & 1.436783 & 0.1045953 \\
\hline 550 & 1.456567 & 0.07080452 \\
\hline 700 & 1.460666 & 0.0864783 \\
\hline 900 & 1.43498 & 0.05895194 \\
\hline \multicolumn{3}{c}{ Parameter $b$} \\
\hline Sample Size & Average Estimate & MSE \\
\hline 400 & 1.5099 & 0.3117199 \\
\hline 550 & 1.596887 & 0.3171107 \\
\hline 700 & 1.542938 & 0.2598158 \\
\hline 900 & 1.509907 & 0.2094074 \\
\hline \multicolumn{3}{c}{ Parameter $\alpha$} \\
\hline Sample Size & Average Estimate & MSE \\
\hline 400 & 1.737238 & 1.683731 \\
\hline 550 & 1.94981 & 2.40539 \\
\hline 700 & 1.772352 & 1.344181 \\
\hline 900 & 1.70319 & 1.563653 \\
\hline \multicolumn{3}{c}{}
\end{tabular}


Appendix C
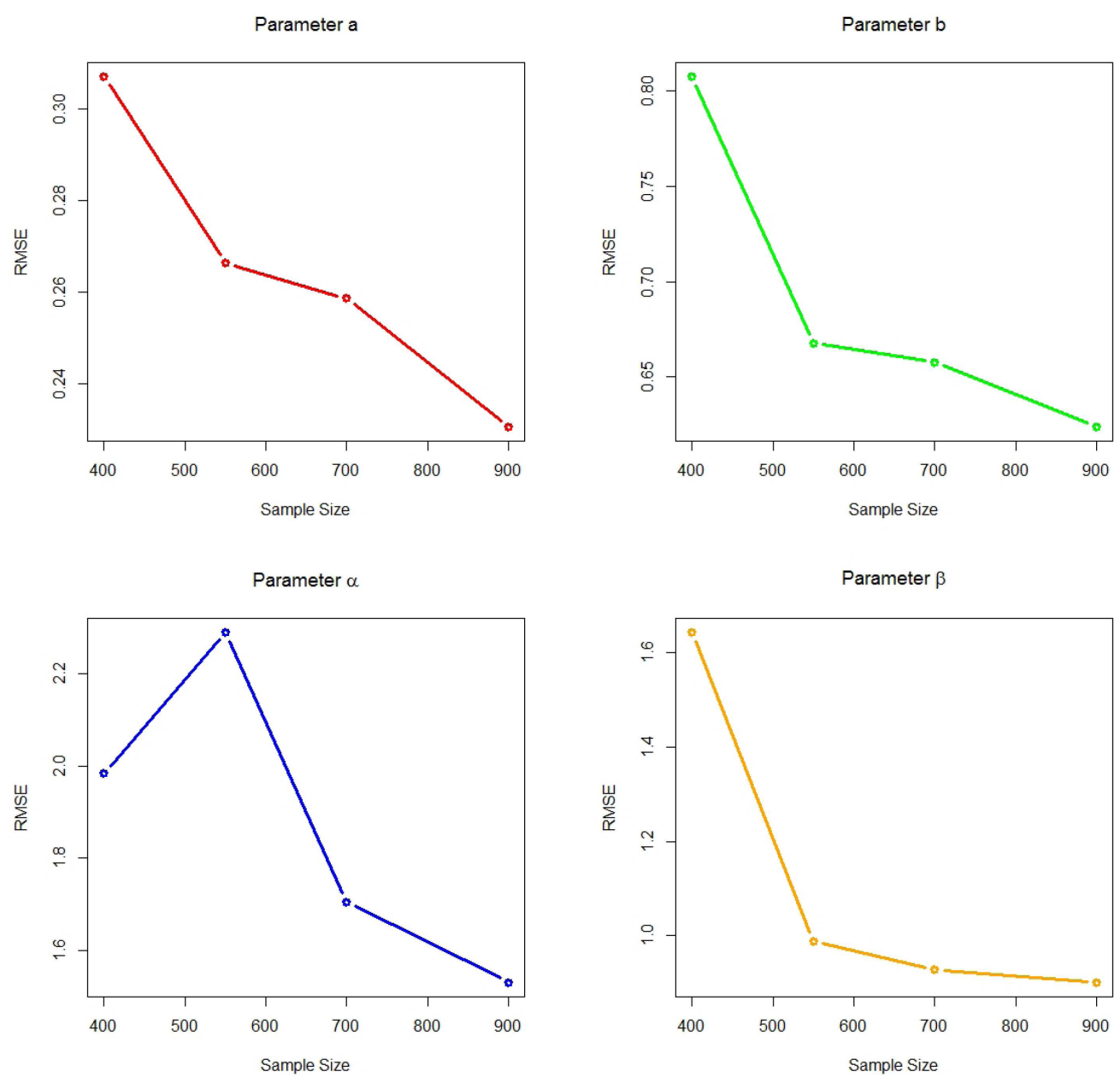

Table 5. Decreasing RMSE for increasing sample size. 


\section{Appendix D}
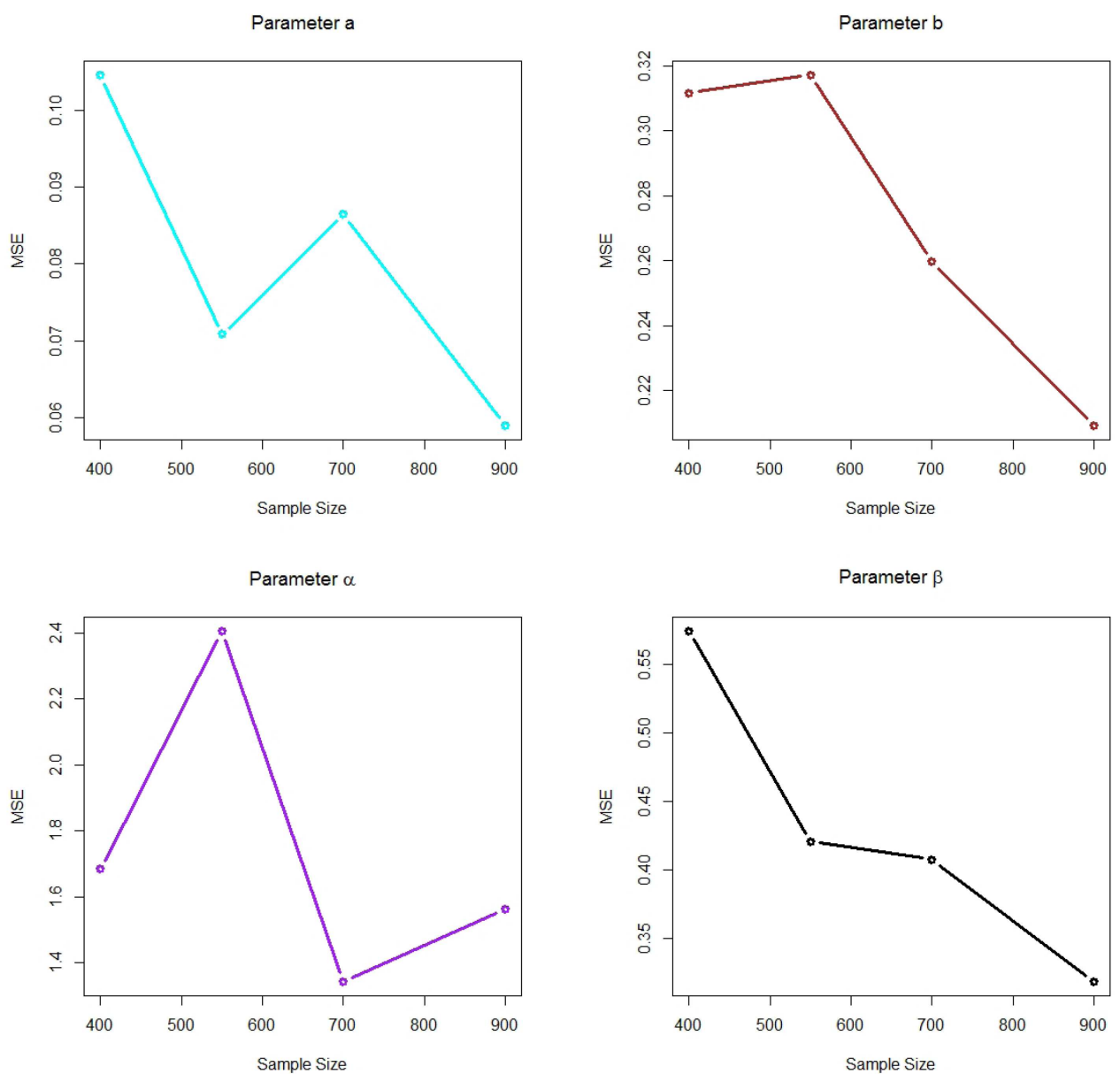

Table 6. Decreasing MSE for increasing sample size. 


\section{Appendix E}
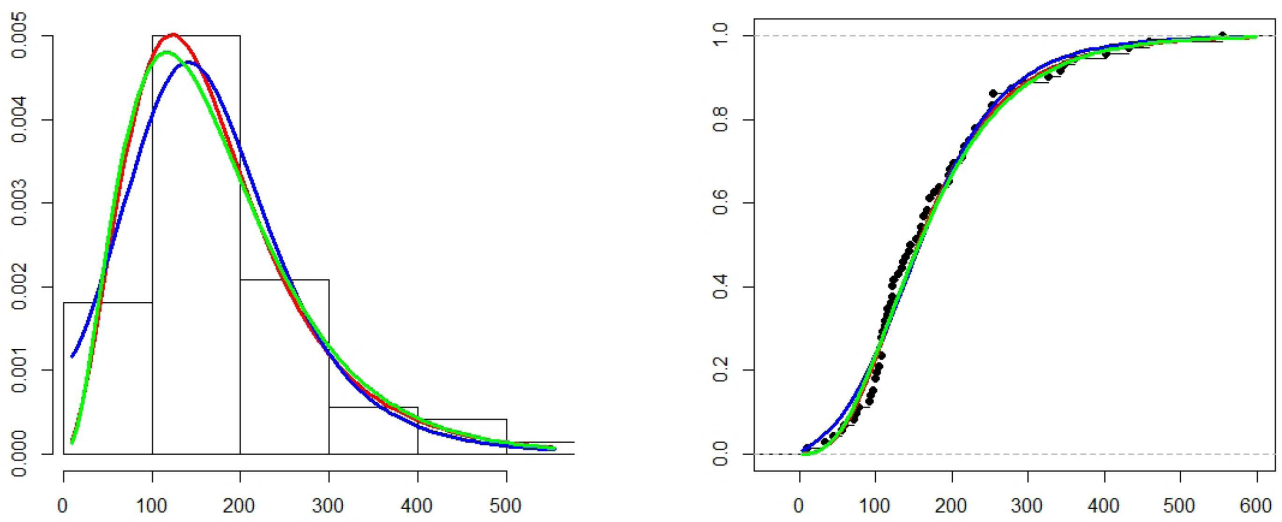

Table 7. Fitted PDFs and CDFs of GAW(red), MOW(blue), and EW(green) to the empirical distribution (right panel) and histogram (left panel) of the guinea pigs data.

\section{Appendix F}

Table 8. Some values of the quantile of the generalized Ampadu-Standard Uniform distribution for some values of $p \in(0,1)$ and choice parameter $\lambda, \beta$.

\begin{tabular}{ccc}
\hline$Q(p)$ & $\lambda=0.4, \beta=2.0$ & $\lambda=1.0, \beta=3.0$ \\
\hline$Q(0.1)$ & 0.2894982 & 0.4026868 \\
$Q(0.2)$ & 0.4129477 & 0.5131957 \\
$Q(0.3)$ & 0.5102535 & 0.5946487 \\
$Q(0.4)$ & 0.5945925 & 0.6630400 \\
$Q(0.5)$ & 0.6710662 & 0.7242429 \\
$Q(0.6)$ & 0.7423060 & 0.7812640 \\
$Q(0.7)$ & 0.8098966 & 0.8359941 \\
$Q(0.8)$ & 0.8748962 & 0.8898470 \\
$Q(0.9)$ & 0.9380681 & 0.9440758
\end{tabular}




\section{Appendix G}

Table 9. The first ten moments of the generalized Ampadu-Standard Uniform distribution for choice parameter $\lambda, \beta$.

\begin{tabular}{ccc}
\hline Moment \# & $\lambda=0.4, \beta=2.0$ & $\lambda=1.0, \beta=3.0$ \\
\hline 1 & 0.6398145 & 0.6954872 \\
2 & 0.4667552 & 0.5250785 \\
3 & 0.3660593 & 0.4180233 \\
4 & 0.3005313 & 0.3453395 \\
5 & 0.2546258 & 0.2931541 \\
6 & 0.22074 & 0.2540699 \\
7 & 0.1947312 & 0.2238155 \\
8 & 0.1741556 & 0.1997674 \\
9 & 0.1574813 & 0.1802329 \\
10 & 0.1437006 & 0.164075 \\
\hline
\end{tabular}

\section{Appendix H}
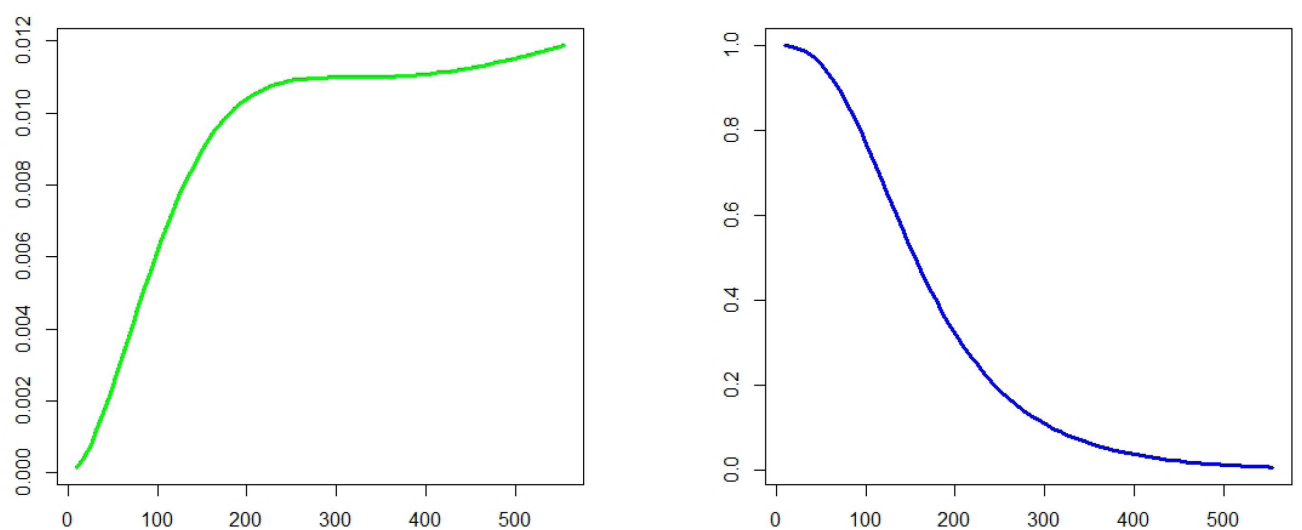

Table 10. The hazard function (left panel) and the survival function(right panel) of the GA-W distribution. 